\title{
Photo-assisted Fabrication of Ribosome Display Microarray
}

\author{
Subhashini Raj Kumal ${ }^{1}$, Ryo Kobayashi ${ }^{1}$, Shingo Ueno ${ }^{1,2}$ and Takanori Ichiki ${ }^{1,2}$ \\ ${ }^{1}$ Department of Bioengineering, School of Engineering, The University of Tokyo \\ 2-11-16 Yayoi, Bunkyo-ku, Tokyo 113-8656, Japan \\ ${ }^{2}$ Core Research of Evolutional Science and Technology, Japan Science and Technology Agency \\ Kawaguchi, Saitama 332-0012, Japan
}

\begin{abstract}
Ribosome display has been widely used for in vitro protein evolution, and the formation of a ternary complex, mRNA-ribosome-protein, is central to this technology as it displays the genotype-phenotype linkage. In conventional ribosome display methods, DNA with a native sequence containing stop codons cannot be used as a starting material as the ribosome in the ternary complex is primarily stalled by the removal of stop codons. Here, a new method for ribosome display is reported. This new method enables the use of DNA containing a stop codon as a starting material and presents the ribosome display on a microarray format with the assistance of a photo-crosslinker. This method is expected to be more advantageous than the conventional method as it allows the use of DNA with a native sequence without any modification, and since it is presented on a microarray format, it enables immediate functional identification and comparison between different proteins without any downstream processing.
\end{abstract}

Keywords: ribosome display, ribosomal stalling, microarray and photo-crosslinker

\section{Introduction}

Ribosome display is an in vitro protein display method that generates and uses mRNA-ribosome-protein ternary complexes for the screening of novel proteins, followed by collection and amplification of the gene encoding protein of interest [1-3]. The ternary complex forms a linkage between genotype (mRNA) and phenotype (protein) in a cell-free system. Ribosome display is more advantageous over other protein display methods, such as phage [4] and plasmid [5] display methods, since it does not require tedious in vivo gene expression (bacterial transformation procedures) to form the genotype-phenotype linkage. Therefore, reactions can be carried out in a cell-free system and in a shorter time. Sidestepping the transformation reaction also allows for larger and more diverse DNA libraries to be used for protein display. Typically, a protein display method that requires in vivo gene expression is limited to a smaller library size $\left(10^{7-10}\right)$ due to the limited capacity of bacterial transformation [6]. In contrast, larger libraries with sizes of $10^{12-14}$ have been used in the ribosome display method [7]. Apart from that, the use of a cell-free system in ribosome display also overcomes the restrictions presented by host cells in cell-based display methods, such as the difficulty in synthesizing toxic or unstable proteins [8].

For a successful ribosome display system, the ribosome of the ternary complex must be stable against dissociation and the displayed protein must be functional. In conventional ribosome display method, the dissociation of the ribosome and the release of the protein and mRNA from the ternary complex is avoided primarily by ribosomal stalling before being further stabilized by decreasing temperature and using highly concentrated magnesium ions. For this purpose, a DNA library modified to remove stop codons is used as the 
starting material $[2,9]$. Naturally occurring stop codon sequences (TAA, TGA, and TAG) present an obstacle to ribosomal stalling and the stabilization of the ternary complex because they are recognized by a group of proteins, known as release factors, which initiate the natural translation termination mechanism causing the release of full-length proteins from ribosome followed by dissociation of the ribosome [10-13]. Functional proteins are formed by the synthesis of full-length proteins followed by folding to form the correct shape. This is achieved by letting the ribosome read and translate the gene encoding the protein of interest completely.

In general, a conventional ribosome display method involves the following steps. A polymerase chain reaction (PCR) fragment that contains a promoter and a gene sequence, which encodes the protein library, without a stop codon is transcribed in vitro to produce mRNA without the stop codon. Cell-free translation is then carried out in a tube using a crude cell-free extract. During translation, the ribosome reads the mRNA until its 3'-terminus to form the mRNA-ribosome-protein ternary complex. Since the mRNA does not have the stop codon, the ribosome stalls once it reaches the end of the mRNA strand and hence displays a full-length protein. The ternary complex is then mixed with the target molecules. The displayed protein of the ternary complex with affinity to the target molecule binds with the target molecule whereas unbound ternary complexes are washed out. From the bound complexes, the mRNAs are collected, reverse transcribed and amplified by PCR to produce the DNA. Several rounds of these steps are carried out to obtain the protein of interest.

The conventional ribosome display method described above has two drawbacks. The first is that it relies heavily on the absence of stop codons in order to stall the ribosomes. Thus, DNA libraries with native sequences that always have stop codons cannot be used as starting molecules for ribosome display. The library must be sequenced first and then modified by PCR to remove the stop codon. In order to use DNA containing the stop codon for ribosome display, Ueda et al. proposed to use an $E$. coli-based reconstituted translation system (PURE system) [14] without release factors to generate the ternary complex. By removing the release factors from the cell-free system, the natural translation termination mechanism can be avoided because now there are no proteins that recognize the stop codon $[15,16]$. Hence, the ribosome is stalled at the stop codon and the ternary complex can be displayed. However, the genetic sequence of DNA used in this method was not native as it was modified to include a glycine/serine spacer sequence and a SecM ribosomal stalling sequence before the stop codon. Another method demonstrated by Aoyama et al. showed that ribosome display using DNA with native sequence containing the stop codon is possible by using an engineered suppressor transfer RNA (tRNA). This method is based on ribosomal read-through where the engineered suppressor tRNA recognizes and enters the ribosome at the stop codon, thus enabling translation to continue without the dissociation of the ternary complex [17]. The DNA used in this work was not modified and the UTR of mRNA was used as a spacer. However, artificial synthesis of the suppressor tRNA is difficult and its incorporation into the ribosome is only efficient in the absence of release factors.

The second drawback is that ternary complexes displaying different proteins are generated in a solution inside a tube and then captured with target molecules either in the solution or on a solid support. Since all the different proteins are mixed and captured together, it is not possible to directly determine which of the captured proteins has the strongest affinity to the target molecule or to compare the binding strength of different proteins without downstream processing. Collection of only one type of protein immediately after binding to the target molecule is also difficult.

Here, a new method of ribosome display is reported in order to overcome the drawbacks associated with the conventional methodologies. First, an unmodified DNA containing the stop codon was used as the starting material, where the stop codon functions to stall the ribosome. 
Secondly, the ribosome display was presented on a microarray format. A microarray consists of miniaturized arrays of biomolecules captured on a solid support and serves as a high-throughput platform for the functional analysis and screening of biomolecules. By presenting the ribosome display on a microarray format, a high-throughput ribosome display platform is formed on a solid support as miniaturization of the spot size allows a large number of ribosome display spots to be formed on a single support. The ribosome display microarray will also enable users to directly detect binding to target molecules and compare the binding strength of the different proteins using fluorescently labeled target molecules. The collection of only one type of protein is also feasible since each spot on the microarray will only display one type of protein.

The ribosome display microarray was fabricated by incorporating microintaglio printing $(\mu \mathrm{IP}) \quad[18,19]$ and a photo-crosslinker, 3-carbovinyl- carbazole $\left({ }^{\mathrm{CNV}} \mathrm{K}\right),[20]$ into the proposed method. With the use of $\mu$ IP, mRNAs containing stop codon were arrayed on a single solid support by hybridization with linker DNA. From the mRNA array, ribosome display array was formed by cell-free translation. The photo-crosslinker, ${ }^{\mathrm{CNV}} \mathrm{K}$, provided a way to permanently link the mRNA to the linker DNA by forming a covalent bond with uridine residue upon photo-irradiation.

\section{Materials and method}

2.1 Preparation of linker DNA and DNA-encoding model protein

Thiol-modified linker DNA with ${ }^{\mathrm{CNV}} \mathrm{K}$, 5'-HS- $\left(\mathrm{CH}_{2}\right)_{6}-\mathrm{TTTTTTTTTTTTTTTTTTTTTTT}$ TTTTTTTTTTTTTTTTTTTTTTTTTTTTGCA- ${ }^{\mathrm{C}}$

${ }^{\mathrm{NV}} \mathrm{K}$-CCGTGTAGTAGTCGC- Spacer 18- Spacer 18- Spacer 18-CC was custom synthesized by Tsukuba Oligo Service, Japan. A DNA construct containing a T7 promoter, a Shine-Dalgarno sequence, a gene encoding green fluorescent protein (GFP) with its stop codon intact, a 6 nucleotides spacer sequence and a hybridizing sequence (for crosslinking with ${ }^{{ }^{C N V}} \mathrm{~K}$ and hybridization with the linker DNA) was used as the model DNA.

\subsection{In vitro transcription of mRNA}

The Cy5-labeled mRNA was transcribed from the DNA encoding the model protein using a T7 RiboMAX Express Large Scale RNA Production System (Promega, USA) and Cy5-UTP (GE Healthcare, UK) in accordance with the manufacturer's protocol.

\subsection{Fabrication of PDMS mold with microwells}

To prepare polydimethylsiloxane (PDMS) mold, SILPOT 184 (Dow Corning Toray Silicone, Japan) and a curing agent (CATALYST SILPOT, Dow Corning Toray Silicone, Japan) were mixed at a ratio of $10: 2$ and $0.8 \mathrm{ml}$ of the mixture was cast onto the SU- 8 master mold and cured for $100 \mathrm{~min}$ at $100^{\circ} \mathrm{C}$. The cured PDMS was peeled off from the master mold and the formation of microwells was confirmed using a light microscope.

\subsection{Fabrication of linker DNA functionalized gold substrate}

Cover glass $(20 \mathrm{~mm} \times 20 \mathrm{~mm} \times 0.5 \mathrm{~mm}$, Matsunami Glass, Japan) was cleaned using a sulfuric acid and hydrogen peroxide mixture (SPM) and subsequently sputter-coated with chrome followed by gold. This gold-coated glass was cleaned using SPM and the surface was functionalized with the thiol-modified linker DNA by incubating with $1 \mu \mathrm{M}$ linker DNA in $1 \times$ phosphate buffered saline (PBS) solution in a sealed chamber for $24 \mathrm{~h}$ at room temperature. Then, the substrate was soaked in $1 \mathrm{mM}$ mercaptohexanol $(\mathrm{MCH})$ in $1 \times$ PBS for $1 \mathrm{~h}$ at room temperature. The substrate was sonicated in $1 \times$ PBS with $0.2 \%$ Tween 20 for 30 min followed by rinsing with $1 \times$ PBS and deionized water. Finally, it was dried using a spin dryer.

\subsection{Microintaglio printing of in vitro transcribed mRNA to form mRNA microarray}

The PDMS mold was sonicated in $10 \mathrm{ml}$ of $0.1 \%$ sodium dodecyl sulfate (SDS) for $3 \mathrm{~min}$ followed by soaking at room temperature for 30 min. The surfactant was used to hydrophilize the PDMS surface. The soaked PDMS was then dried 
on a flat plate thermal cycler at $80^{\circ} \mathrm{C}$ for $1 \mathrm{~min}$. After drying, the temperature of the thermal cycler was then reduced to $4^{\circ} \mathrm{C}$ and $1 \mu \mathrm{M}$ Cy5-labeled mRNA in $3 \times$ saline sodium citrate (SSC) and $0.02 \%$ SDS was added onto the PDMS and spread with a pipette tip. The PDMS was sealed with the linker DNA functionalized gold substrate using 2 $\mathrm{kg}$ of weight. The temperature of the thermal cycler was then increased to $70^{\circ} \mathrm{C}$ at a ramp rate of $1{ }^{\circ} \mathrm{C} / \mathrm{s}$ and held for $30 \mathrm{~min}$ to denature the mRNA, after which it was cooled to $25^{\circ} \mathrm{C}$ at a ramp rate of $0.1^{\circ} \mathrm{C} / \mathrm{s}$ and held for $10 \mathrm{~min}$ to hybridize the mRNA with the linker DNA. After hybridization, the gold substrate was washed once in $3 \times \mathrm{SSC}$, $0.1 \times$ SSC with $0.1 \%$ SDS, and $0.1 \times$ SSC solution for 5 min each.

\subsection{Photo-crosslinking of ${ }^{\mathrm{CNV}} \mathrm{K}$ with residue of mRNA}

$100 \mu 1$ of $0.1 \times$ SSC solution was added to the mRNA array. The ${ }^{\mathrm{CNV}} \mathrm{K}$ molecule on linker DNA was crosslinked with the uridine residue on mRNA by exposing the substrate to $365 \mathrm{~nm}$ ultraviolet (UV) light for $30 \mathrm{~s}$. Figure 1 shows the formation of the covalent bond during this photo-crosslinking. Partial crosslinking of the substrate was achieved by partially covering the substrate with either black

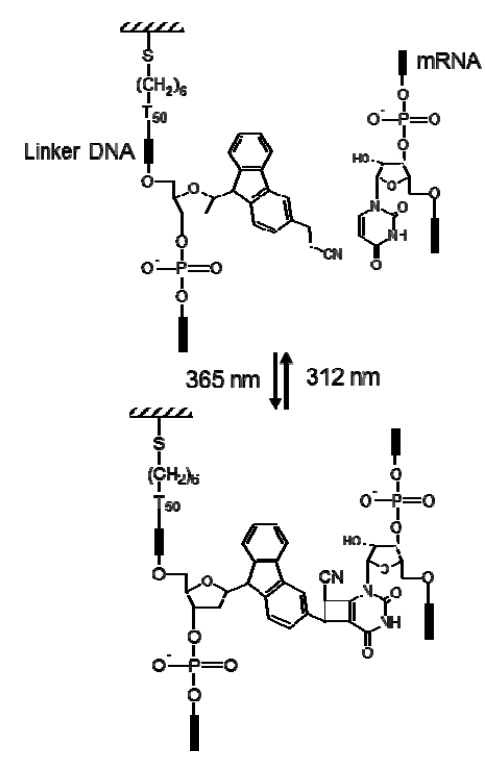

Figure 1: Reversible photo-crosslinking between ${ }^{\mathrm{CNV}} \mathrm{K}$ of linker DNA and uridine residue of mRNA. sugar paper or aluminum foil before exposing it to UV light. After photo-crosslinking, the mRNA array in $0.1 \times$ SSC buffer was imaged using a confocal laser scanning microscope (Carl Zeiss LSM 510) at $633 \mathrm{~nm}$ with an APD detector.

\subsection{On-chip protein synthesis and fabrication of ribosome display microarray}

An E. coli-based reconstituted translation system without release factors (PURExpress, New England Biolabs, USA) was used as the cell-free protein synthesis system. $65 \mu 1$ of cell-free protein synthesis mix containing FluoroTect Green Lys (lysine-charged tRNA labeled with BODIPY FL, Promega) was added onto the mRNA array chip and incubated in a sealed chamber at $37^{\circ} \mathrm{C}$ for 40 min. Translation reaction was stopped by cooling the substrate on ice, and a solution of $50 \mathrm{mM}$ magnesium acetate and $150 \mathrm{mM}$ potassium chloride was sprayed onto the substrate to stabilize the mRNA-ribosome-protein complex. The solution was exchanged several times to remove excess protein synthesis mix and FluoroTect Green Lys. This ribosome display array was then imaged using the confocal laser scanning microscope. Protein and mRNA were visualized using an APD detector at Ex/Em: $488 \mathrm{~nm} / \mathrm{LP} 505$ and $633 \mathrm{~nm} / \mathrm{BP}$ 655-710 IR, respectively.

\section{Results and discussion}

Figure 2 outlines the procedure for the fabrication of the ribosome display microarray. Thiol-modified linker DNA with ${ }^{\mathrm{CNV}} \mathrm{K}$ was immobilized onto the gold substrate by thiol-gold binding. The unreacted gold surface was then blocked with mercaptohexanol (MCH). The DNA encoding the model protein with the stop codon was transcribed in vitro in the presence of Cy5-UTP to produce Cy5-labeled mRNA with the stop codon. Using PDMS with microwells, the mRNA was then $\mu$-intaglio printed onto the gold surface and hybridized with linker DNA to form the mRNA microarray. To form a covalent bond between the mRNA and the linker DNA, the mRNA hybridized surface was irradiated with ultraviolet light at 365 $\mathrm{nm}$, resulting in crosslinking between the ${ }^{\mathrm{CNV}} \mathrm{K}$ of linker DNA and the uridine residue of mRNA. 


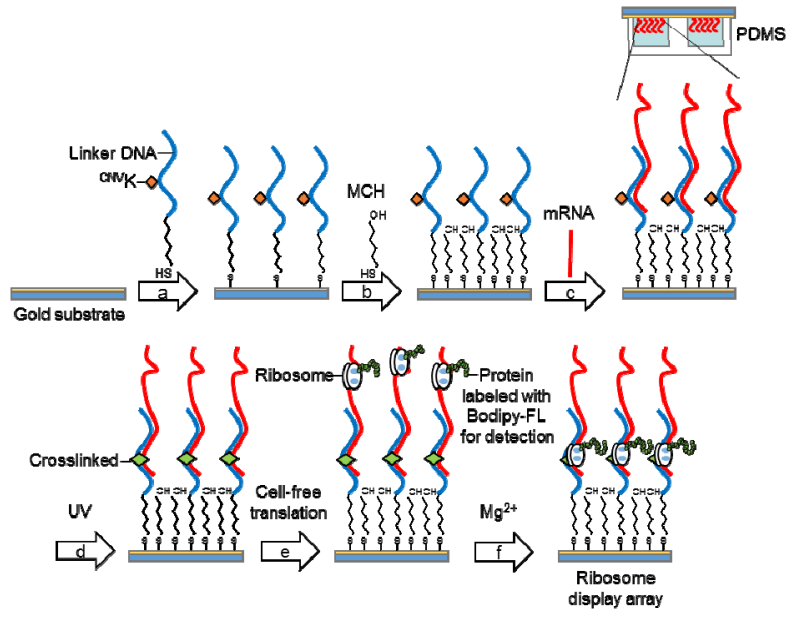

Figure 2: Fabrication of ribosome display microarray using $\mu$-intaglio printing and a photo-crosslinker involves (a) immobilization of linker DNA containing ${ }^{\mathrm{CNV}} \mathrm{K}$ on a gold surface through gold-thiol binding, (b) blocking of unreacted gold surface with mercaptohexanol (MCH), (c) printing of in vitro transcribed mRNA using PDMS with microwells, (d) photo-crosslinking at $365 \mathrm{~nm}$ to form covalent bonds, (e) cell-free translation using the PURE system without release factors, and (f) stalling of the ribosome by the addition of magnesium-rich buffer to avoid dissociation of the ribosome.

Translation was started by adding the PURE system in the presence of FluoroTect Green Lys. FluoroTect Green Lys labels the synthesized protein with Bodipy-FL to aid in visualization during imaging. During translation, each mRNA strand is read by several ribosomes and each ribosome can be found at different positions on the mRNA. The ribosome stalls once it reaches the stop codon because there are no release factors in the cell-free system. In this work, the translation reaction was carried out for a few minutes and was stopped by cooling the reaction on ice with the addition of a buffer containing high concentrations of magnesium ions. At this stage, the ribosomes were stalled in their current position as the ternary complex and were stabilized against dissociation by the highly concentrated magnesium ions.

Figure 3 shows the results of the above procedure. PDMS with microwells of $60 \mu \mathrm{m}$ diameter was used to print and hybridize the mRNA onto the gold substrate. Part of the mRNA microarray was irradiated with ultraviolet light to form a photo-crosslinked and non-crosslinked region on the same surface. The mRNA array was imaged after mRNA hybridization and photo-crosslinking, whereas the ribosome display array was imaged after the translation reaction and addition of magnesium-rich buffer. In the area that was not photo-crosslinked, the fluorescence intensity of Cy5-labeled mRNA decreased greatly after translation whereas the fluorescence of Bodipy-FL-labeled protein was not visible. In the area that was photo-crosslinked, both the Cy5-labeled mRNA and Bodipy-FL-labeled protein were successfully visualized after the translation reaction, indicating the successful generation of the ternary complex.

The decrease in the fluorescence intensity of Cy5-labeled mRNA, which was only observed in the non-crosslinked area, after translation indicates the loss of hybridized mRNA. This loss could be attributed to the absence of the photo-crosslinked covalent bond and its mechanism can be explained as follows. Since there are no release factors in the cell-free system, the ribosome stalls once it reaches the stop codon. At this point, ribosomal read-through [17] occurs when aminoacylated

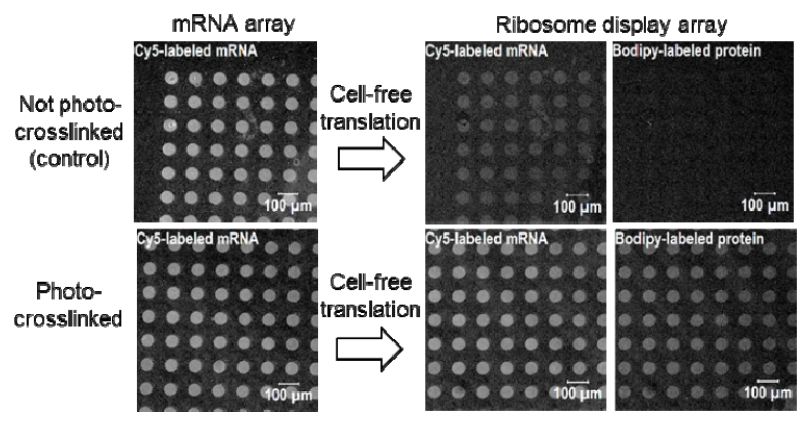

Figure 3: Results of on-chip ribosome display fabricated using $\mu$-intaglio printing and photo-crosslinker. Only mRNA that was photo-crosslinked with ${ }^{\mathrm{CNV}} \mathrm{K}$ resulted in a ribosome display. In areas without crosslinking, the protein is released immediately after synthesis followed by the dissociation of the ribosome as there is no covalent bond to stall the ribosome. The diameter of spots is $60 \mu \mathrm{m}$. Imaging was carried out using a confocal laser scanning microscope. 
tRNA with similar anticodons to the stop codon enters the ribosome causing the ribosome to continue translating the mRNA beyond the stop codon. Once the ribosome reaches the hybridized region, it starts to break the non-covalent hydrogen bonds between the mRNA and the linker DNA by acting as mRNA helicase [21]. When this occurs repeatedly, the mRNA will be released from the hybridization and subsequently from the gold surface, leading to the decrease in the fluorescence intensity of Cy5-labeled mRNA.

The difference in the fluorescence intensities of the Bodipy-FL-labeled protein between the non-crosslinked area and the crosslinked area confirms that ribosome display was aided by the photo-crosslinking generated covalent bond. Ribosome display is not possible in the area that was not photo-crosslinked because ribosomal read-through releases the protein and mRNA from the surface. In the area that was photo-crosslinked, the ternary complex can be saved from dissociation because the ribosome cannot break the ${ }^{\mathrm{CNV}} \mathrm{K}$-assisted covalent bond. Hence, the covalent bond formed by photo-crosslinking ${ }^{\mathrm{CNV}_{\mathrm{K}}}$ functioned not only as a means to avoid the loss of mRNA from the array during translation, but also as an additional stalling mechanism in the event of ribosomal read-through.

Figure 4 shows that by using $\mu$-intaglio printing, a high-throughput ribosome display microarray can be realized by reducing the spot size to $6 \mu \mathrm{m}$. PDMS with microwells of $6 \mu \mathrm{m}$ thickness and $100-6 \mu \mathrm{m}$ diameter was used to print and hybridize the mRNA onto the gold substrate. Figure 4(a) shows the scanned image while Figure 4(b) shows the quantified data of 4(a). Quantification of the mRNA array indicates that the fluorescence intensity decreases with spot size. This decrease can be attributed to the adsorption of mRNA molecules onto the PDMS during $\mu$-intaglio printing, which decreases the number of mRNA molecules available for printing. This effect is more pronounced in smaller wells since the number of mRNA molecules left for hybridization after adsorption is small in comparison with larger wells where the effect of adsorption on mRNA printing is less pronounced. Quantification of the ribosome

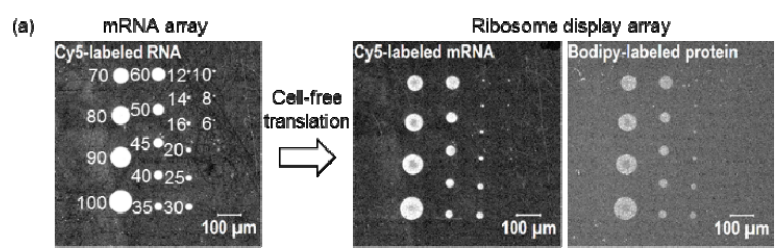

(b)
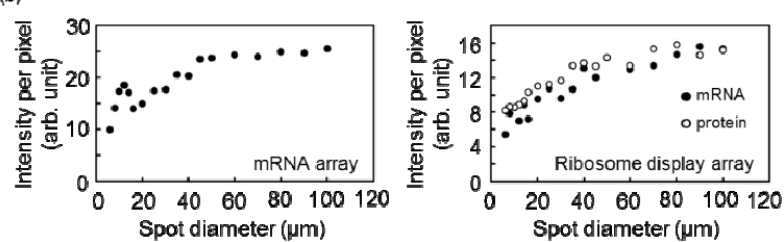

Figure 4: Results of on-chip ribosome display fabricated using $\mu$-intaglio printing at various spot sizes ranging from 100-6 $\mu \mathrm{m}$. (a) Scanned images show that both the mRNA array and ribosome display array were successfully fabricated. The fluorescence intensities of hybridized mRNA and synthesized protein decrease with spot size. (b) Quantified results of (a) with closed circles representing the fluorescence intensity of Cy5-labeled mRNA and open circles representing the fluorescence intensity of Bodipy-FL-labeled protein. Imaging was carried out using a confocal laser scanning microscope. Quantification was accomplished using ImageJ software (NIH, USA). Intensity-per-pixel values are the mean gray values generated by dividing the sum of gray values of all the pixels in the selected area with the number of pixels.

display array indicates that the amount of protein decreases with spot size, and this decrease is parallel to the decrease in the amount of hybridized mRNA. This is because the decrease in the number of hybridized mRNA reduces the number of proteins displayed.

In the conventional ribosome display method, the protein of interest is usually selected through its affinity to the target molecules. In contrast, the ribosome display microarray fabricated using the proposed method can be applied beyond the characteristics of binding affinity. It can be widely applied in the screening and analysis of novel proteins, similar to a protein array, and enables the direct retrieval of mRNAs for DNA synthesis through the reversible photo-crosslinking of ${ }^{\mathrm{CNV}} \mathrm{K}$ to obtain the genetic information. In future work, a spacer sequence of at least 23-26 amino acids in length will be added to the DNA after the stop 
codon. The spacer sequence will be added to ensure the synthesized protein is out of the ribosome tunnel $[22,23]$ and is able to fold without losing its physical linkage with the ternary complex since the spacer sequence will occupy the ribosome tunnel. Since the modification of DNA to add the spacer sequence does not change the native genetic sequence and does not require prior knowledge of the DNA sequence, a DNA library with native sequence can be used with the proposed method in addition to the spacer sequence to fabricate the ribosome display microarray.

\section{Conclusion}

The ribosome display microarray was successfully fabricated by employing $\mu \mathrm{IP}$ and photo-crosslinking using DNA containing stop codon as the starting material. $\mu$ IP enabled miniaturization of the ribosome display spot size while the photo-crosslinking assisted covalent bond circumvented the loss of mRNA from the array during translation and functioned as an additional stalling mechanism. The demonstrated method allows the use of DNA with a native sequence containing stop codon as starting material and presents the ribosome display on a microarray format which enables direct functional identification and comparison of different proteins without any downstream processing.

\section{References}

1. L. C. Mattheakis, R. R. Bhatt and W. J. Dower, Proc. Natl. Acad. Sci. USA, 91 (1994) 9022

2. J. Hanes and A. Pluckthun, Proc. Natl. Acad. Sci. USA, 94 (1997) 4937

3. J. Hanes, L. Jermutus and A. Pluckthun, Methods Enzymol., 328 (2000) 404

4. G. P. Smith and V. A. Petrenko, Chem. Rev., 97 (1997) 391

5. R. E. Speight, D. J. Hart, J. D. Sutherland and J. M Blackburn, Chem. Biol., 8 (2001) 951
6. M. He and F. Khan, Expert Rev. Proteomics, 2 (2005) 421

7. T. Lamla and V. A. Erdmann, J. Mol. Biol., 329 (2003) 381

8. A. M. Jackson, J. Boutelle, N. Cooley and M. He, Brief. Funct. Genomic Proteomic, 2 (2004) 308

9. M. He and M. J. Taussig, Nucleic Acids Res., 25 (1997) 5132

10. L. Kisselev, M. Ehrenberg and L. Frolova, EMBO J., 22 (2003) 175

11. Y. Nakamura and K. Ito, Trends Biochem Sci., 28 (2003) 99

12. M. Y. Pavlov, A. Antoun, M. Lovmar and M. Ehrenberg, EMBO J., 27 (2008) 1706

13. E. M. Youngman, M. E. McDonald and R. Green, Annu. Rev. Microbiol., 62 (2008) 353

14. Y. Shimizu, A. Inoue, Y. Tomari, T. Suzuki, T. Yokogawa, K. Nishikawa and T. Ueda, Nat. Biotechnol., 19 (2001) 751

15. H. Ohashi, Y. Shimizu, B. W. Ying, T. Ueda, Biochem. Biophys. Res. Comm., 352 (2007) 270

16. T. Ueda, T. Kanamori and H. Ohashi, Methods Mol. Biol., 607 (2010) 219

17. A. Ogawa, S. Sando and Y. Aoyama, Chem. Bio. Chem., 7 (2006) 249

18. M. Biyani, T. Osawa, N. Nemoto and T. Ichiki, Appl. Phys. Express, 4 (2011) 047001

19. M. Biyani, J. Moriyasu, Y. Tanaka, S. Sato, S. Ueno and T. Ichiki, Appl. Phys. Express, 6 (2013) 087001

20. Y. Yoshimura and K. Fujimoto, Org. Lett., 10 (2008) 3227

21. S. Takyar, R. P. Hickerson and H. F. Noller, Cell, 120 (2005) 49

22. H. Nakatogawa and K. Ito, Cell, 108 (2002) 629

23. A. Schaffitzel and N. Ban, J. Struct. Biol., 158 (2007) 463 